\title{
O Papel da Função Reforçadora das Relações Verbais de Tato e Textual, em Contexto de Equivalência de Estímulos, em Universitários
}

\author{
The Role of Reinforcing Function of Tact and Textual Verbal Relations \\ in the Context of Stimulus Equivalence, in College Students
}

\author{
Luiza de Moura Guimarães*, Giovana Escobal \& Celso Goyos \\ Universidade Federal de São Carlos, São Carlos, São Paulo, Brasil
}

\begin{abstract}
Resumo
A aprendizagem das relações verbais de tato podem apresentar características de emergência e manutenção diferentes das relações textuais. Essas diferenças despertam particular interesse quando os estímulos que controlam tais operantes verbais pertencem à mesma classe de equivalência e a topografia da resposta é semelhante. O objetivo deste trabalho foi investigar o papel da função reforçadora de cada uma dessas relações na emergência de novas relações. Inicialmente, seis universitários participaram do estudo. Primeiro, foram ensinadas, através de tarefas de matching-to-sample, relações de ouvinte entre palavras ditadas e figuras, e entre palavras ditadas e palavras impressas. Após ensino, foram testadas as relações de equivalência, e a emergência de relações de tato e textual-consideradas relações de falante. Em seguida, os participantes foram submetidos a esquemas concorrentes com encadeamento para verificar a preferência por tarefas. Os resultados indicaram indiferença na preferência por tarefas, o que é consistente com o estabelecimento das relações de equivalência e com o fato dos participantes demonstrarem desempenho similar nos testes de tato e leitura. A ausência de preferência pode ter sido observada em função da indiferença da função reforçadora desses operantes, mas também pelo fato dos participantes terem um vasto e sofisticado repertório verbal.

Palavras-chaves: Comportamentos emergentes, função reforçadora do estímulo, escolha, preferência, tato, textual.
\end{abstract}

\begin{abstract}
Learning tact verbal relations may present characteristics of emergence and maintenance different from learning textual relations. These differences arouse particular interest when the stimuli that control such verbal operant belong to the same equivalent class and the response topography is similar. The objective of this study was to investigate the role of reinforcing function of each of these relationships in the emergence of new relations. Initially, six students participated in the study. First, we taught, through matching-to-sample tasks, listener relations between dictated words and pictures, and between dictated words and printed words. After training, we tested equivalence relations, and the emergence of tact and textual relations which are considered speaker relations. Then, the participants were submitted to concurrent schedules of reinforcement to evaluate preference for tasks. The results showed indifference in preference for tasks, which is consistent with the establishment of equivalence relations and with the fact that participants show similar performance on tact and textual tests. The absence of preference can be observed because of the indifference in those operant reinforcing functions, but also, because of the vast and sophisticated verbal repertoire of the participants.

Keywords: Emerging behavior, reinforcing function of stimuli, choice, preference, tact, textual.
\end{abstract}

\footnotetext{
"Endereço para correspondência: Laboratório de Aprendizagem Humana, Multimídia Interativa, \& Ensino Informatizado, Departamento de Psicologia, Universidade Federal de São Carlos, Via Washington Luís, km 235 , Caixa Postal 676, São Carlos, SP, Brasil 13565-905. E-mail: luizademoura@hotmail.com, giovanaescobal@ hotmail.com e celsogoyos2@gmail.com.

Os autores agradecem o apoio da FAPESP por bolsa MS a LMG (Proc. 2010/14525-0) e bolsa PD a GE (Proc. 2010/11201-9), e ao CNPq por bolsa produtividade em pesquisa a CG. Os autores também agradecem pelos comentários, críticas e sugestões de Nassim Chamel Elias em versões anteriores do presente manuscrito.
}

Nos estudos sobre equivalência de estímulos, o procedimento básico mais frequentemente empregado consiste em apresentações sucessivas da tarefa de escolha de acordo com o modelo, $\mathrm{MTS}^{1}$, e é utilizado para ensinar relações entre estímulos através de discriminações condicionais e para testar relações emergentes entre esses estímulos. Uma discriminação condicional é definida como uma discriminação de segunda ordem, na qual uma resposta para um 
estímulo discriminativo é diferencialmente consequenciada somente na presença de um outro estímulo, o estímulo condicional. O pressuposto desse modelo experimental, elaborado em Sidman e Tailby (1982), é de que uma vez ensinadas diretamente relações entre estímulos de dois ou mais conjuntos - (relações entre estímulos dos conjuntos $\mathrm{A}$ e B, e A e C, por exemplo), outras relações entre estímulos emergem sem ensino direto - (por exemplo, relações entre estímulos dos conjuntos B e A, C e A, B e C, e C e $B)$. No momento em que qualquer um dos estímulos que pertencem às relações ensinadas é substituído por outro, sem alterar a probabilidade da ocorrência da resposta, diz-se que os estímulos correspondentes dos três conjuntos (A, B e C) formam uma mesma classe, os estímulos são equivalentes ou mutuamente substituíveis, e dentro da mesma classe têm a mesma função para o organismo (Elias, Goyos, Saunders, \& Saunders, 2008; Rodríguez-Mori \& Pérez-González, 2005).

Sidman e Tailby (1982), com base em conceitos derivados da matemática, sugerem que as relações entre os estímulos devem apresentar algumas propriedades empiricamente testáveis para que sejam considerados equivalentes. São essas as propriedades de reflexividade, simetria e transitividade, apresentadas em condições de testes - sem reforço programado - e a partir do ensino das relações condicionais fundamentais. A propriedade de reflexividade implica que cada um dos estímulos das relações ensinadas deve apresentar relação de igualdade com ele mesmo (A-A, B-B, C-C). A propriedade de simetria é inferida a partir da emergência do inverso de uma relação treinada diretamente. Se as relações A-B e A-C são ensinadas diretamente, então espera-se, por simetria, a emergência das relações B-A, e C-A. A propriedade de transitividade é inferida quando, a partir das mesmas condições de ensino, as relações B-C e C-B, emergem sem necessidade de ensino direto específico para elas (Sidman $\&$ Tailby, 1982). Para uma visão expandida e atualizada do conceito de equivalência de estímulos referimos o leitor a Sidman (1994, 2000).

Pesquisas têm demonstrado o desenvolvimento de uma tecnologia poderosa derivada do conceito e procedimentos de equivalência de estímulos, principalmente com aplicações na área de ensino para indivíduos com desenvolvimento típico (Critchfield \& Fienup, 2010; Fienup, Covey, \& Critchfield, 2010; Stromer, Mackay, \& Stoddard, 1992) e com deficiência intelectual (Escobal, Rossit, \& Goyos, 2010; Rossit \& Goyos, 2009).

Estudos que se preocupam também em ampliar a noção comportamental de linguagem para além do arcabouço da equivalência de estímulos, tais como, para investigar a emergência de alguns dos operantes verbais descritos por Skinner (1957), ou o comportamento de nomeação (Horne, Hughes, \& Lowe, 2006). Por exemplo, Ribeiro, Elias, Goyos e Miguel (2010) utilizaram tarefas de MTS para ensinar a relação de tato e para testar as relações de mando.

Diferenças entre os desempenhos nas relações de tato (emitir uma resposta verbal, por exemplo, falar na presença de uma figura) e relações textuais (emitir uma resposta verbal, palavra falada, na presença de uma palavra impressa) têm sido apontadas pela literatura (Elias et al., 2008; Sidman, 1971; Sidman \& Cresson, 1973; Sidman, Cresson, $\&$ Willson-Morris, 1974), embora a topografia da resposta seja praticamente a mesma e os estímulos pertencerem à mesma classe de equivalência. Segundo Sidman (1994), dizer que membros de uma mesma classe diferem entre si é uma contradição, no entanto, alguns estudos apontam para variações quantitativas nas relações de equivalência.

No estudo de Sidman (1971), um garoto de 17 anos, institucionalizado, e com deficiência intelectual tateou todas as 20 figuras familiares (100\%), mas o desempenho no comportamento textual do nome escrito dessas figuras foi de aproximadamente $75 \%$, após estabelecimento das classes de equivalência. No estudo mais recente de Elias et al. (2008) com sete participantes adultos com deficiência intelectual, quatro desses também surdos, eram solicitados a fazer sinais na presença de figuras familiares e de suas palavras impressas correspondentes. Apenas um participante não foi submetido ao teste de tato. $\mathrm{O}$ resultado do estudo mostrou que o desempenho de 4 dos 6 participantes era $40 \%$ mais acurado quando a sinalização foi realizada frente a figura $(22 \%, 29 \%, 55 \%$ e $56 \%)$ do que frente a palavra impressa, apesar dos estímulos terem sido previamente estabelecidos como pertencentes a uma mesma classe de estímulos equivalentes. Em Sidman e Cresson (1973) com dois adolescentes com Síndrome de Down, o participante B.A. apresentou desempenhos de aproximadamente $95 \%$ para tato e $90 \%$ para textual e o participante J.W. apresentou desempenhos aproximados de $75 \%$ para tato e $65 \%$ para textual no último teste realizado, após ensino de todas as relações. Em Sidman et al. (1974) com dois adolescentes com Síndrome de Down, somente um foi submetido aos testes e obteve desempenho aproximado de $50 \%$ de acerto nas relações textuais comparativamente a $70 \%$ de acerto nas relações de tato no último teste realizado.

Uma possibilidade para explicar a diferença no desempenho apontado é que a história de vida do organismo com uma particular relação tenha sido diferente da outra. Em uma tentativa inicial de se colocar a questão sob a ótica da análise comportamental, é possível que haja diferenças nos valores reforçadores dessas tarefas. Se essa interpretação for verdadeira, o estabelecimento de classes de equivalência envolvendo um conjunto de estímulos auditivos e dois conjuntos de estímulos visuais, um de imagens e outro de palavras impressas, unidos pelo nódulo dos estímulos auditivos em classes de equivalência poderá produzir desempenho semelhante ou diferenciado nas relações emergentes de comportamento de tato e de comportamento textual. Se os estímulos forem equivalentes, e o desempenho semelhante, então os desempenhos nos testes de preferência serão indiferentes. Se os desempenhos forem diferentes, então os testes de preferência mostrarão preferência por uma das tarefas, a de comportamento de tato, ou a de comportamento textual.

Uma maneira de acessar preferências poderia envolver o fornecimento de repetidas oportunidades de escolha entre duas ou mais alternativas, simultaneamente disponíveis, para 
se determinar qual é escolhida mais frequentemente (Escobal \& Goyos, 2008). Dentre os procedimentos mais utilizados para estudar o comportamento de escolha estão os esquemas concorrentes com encadeamento. Esquemas concorrentes com encadeamento envolvem a apresentação simultânea de duas ou mais condições de estímulo associadas, cada uma delas, a um esquema específico de reforçamento concorrentemente disponíveis. Cada uma dessas condições é chamada de elo inicial, analogia aos elementos de uma corrente ou cadeia. Uma vez que o indivíduo atende ao esquema de um elo inicial, uma outra condição é apresentada como consequência, referida como elo terminal. Enquanto o indivíduo responde sob o esquema de um elo inicial, o outro continua presente na situação, podendo o indivíduo responder sob o esquema associado a este outro elo. Quando o esquema em vigor no elo terminal for concluído, a consequência programada é apresentada. Esse procedimento separa a eficácia reforçadora do elo terminal das contingências que mantêm o responder neste elo (Catania, 1999).

Com base nessas considerações, o objetivo do presente estudo foi investigar o valor reforçador do estímulo figura e palavra impressa, após o estabelecimento das classes, e se esse elemento se correlaciona com os desempenhos de tato e leitura dos indivíduos na presença desses estímulos.

\section{Método}

\section{Participantes}

Participaram do estudo seis alunos universitários, três do gênero feminino (Irene, Larissa e Nara) e três do gênero masculino (Gabriel, Lucas e Ricardo), com idades entre 22 e 27 anos. Todos recrutados a partir de anúncio em campus universitário de uma cidade do interior paulista. O critério para a participação era de que os participantes não pertencessem ao Curso de Graduação em Psicologia e não tivessem experiências com procedimentos de MTS.

\section{Local, Ambiente Experimental e Materiais}

O estudo foi realizado no próprio campus universitário em que os participantes foram recrutados, e o ambiente experimental montado em uma sala de aproximadamente $4 \mathrm{~m} \times 3 \mathrm{~m}$, contendo duas cadeiras, uma mesa, um microcomputador com monitor colorido, kit multimídia e uma câmara filmadora para a gravação de dados. O programa MestreLibras (Elias \& Goyos, 2010) foi utilizado para programar as sessões, realizar a apresentação dos estímulos e das consequências, registrar e armazenar os dados das sessões experimentais. Nas avaliações de preferências foi utilizado o programa Microsoft PowerPoint 2007 e um protocolo de registro observacional. Uma filmadora montada sobre um tripé em um canto da sala foi utilizada para posterior avaliação de um segundo observador. Em todas as sessões, apenas o participante e a experimentadora estavam presentes na sala.

\section{Estímulos Experimentais}

Foram utilizados três conjuntos de estímulos abstratos com três elementos cada um. O primeiro conjunto (Conjunto A) constituiu-se de estímulos auditivos, palavras faladas formadas por consoante, vogal, consoante, vogal e foram apresentados simultaneamente com um quadrado branco de $8 \mathrm{~cm}$ de lado, na metade superior da tela do computador. O Conjunto B constituiu-se de estímulos visuais, figuras abstratas em preto e branco retiradas do estudo de Hayes, Kohlenberg e Hayes (1991) e o Conjunto C constituiu-se de estímulos visuais, palavras impressas formadas por consoante, vogal consoante, vogal, formatados em fonte Times, cor preta, tamanho 110 . O conjunto A' constituiu-se de respostas orais emitidas pelo participante, correspondentes aos estímulos do conjunto $\mathrm{A}$, e se referiam aos operantes de tato e textual. A Tabela 1 apresenta os estímulos utilizados no estudo.

\section{Tabela 1}

Estímulos Experimentais Utilizados: Palavras Faladas, Figuras e Palavras Impressas

\begin{tabular}{cccc}
\hline Classes/ & Palavras & Figuras & Palavras \\
Conjuntos & faladas & & Impressas
\end{tabular}

1 "VEKI"

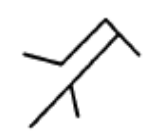

VEKI

2 "JUXO"

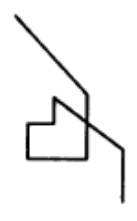

JUXO

\section{Procedimentos Preliminares}

Antes da realização dos procedimentos gerais foram desenvolvidos dois pré-testes.

Avaliação de Preferência por Figuras e Palavras Impressas. Para estabelecimento da hierarquia de estímulos experimentais de preferência dos participantes, realizou-se um teste de preferência por figuras e por palavras impressas. O comportamento de escolha foi observado sob esquemas de reforçamento concorrentes com encadeamento. O participante tinha à sua frente dois botões na mesma cor vermelha, Botão 1 (lado esquerdo) e Botão 2 (lado direito). Ao início de cada tentativa, o participante recebia a instrução: "Aponte qual você quer". Nos elos iniciais, respostas no Botão 1 em esquemas de 
razão fixa 1 (FR1) foram seguidas da apresentação de um dos estímulos do Conjunto B, uma figura, em um fundo branco (Alternativa 1), no respectivo elo terminal. Respostas no Botão 2 em esquemas de razão fixa 1 (FR1) foram seguidas da apresentação de um dos estímulos do Conjunto C, uma palavra, um fundo branco (Alternativa 2 ), no respectivo elo terminal. Os três estímulos dos Conjuntos $\mathrm{B}$ e os três estímulos do Conjunto $\mathrm{C}$ foram apresentados alternadamente após respostas nos Botões 1 e 2 , respectivamente.

A posição das alternativas nesse contexto era fixa. Após a apresentação do estímulo por 2 segundos, uma nova tentativa era apresentada. Uma sessão era composta por 20 tentativas. O critério para estabilidade de respostas de escolha e término dessa fase foi definido como a emissão de $75 \%$ ou mais respostas de escolha nos elos iniciais dos esquemas com preferência para uma das alternativas em duas sessões consecutivas. Quando o participante atingia o critério para desempenho nessa fase, a posição das alternativas: figura em fundo branco e palavra impressa em um fundo branco era invertida (Caso o participante demonstrasse preferência por um dos tipos de estímulos, figura - Conjunto B - ou palavra impressa -Conjunto C -, a apresentação dos Conjuntos $\mathrm{B}$ e $\mathrm{C}$ eram invertidas para os botões 2 e 1 , respectivamente).

Respostas Orais diante das Figuras e Palavras Impressas. Cada tarefa desse tipo iniciou com a apresentação de um estímulo modelo, centralizado na metade superior do monitor do computador e da instrução "toque". Quando o participante tocava no estímulo apresentado, a instrução "fale o nome" era fornecida. Todas as respostas orais dos participantes foram seguidas pela apresentação imediata de uma nova tentativa. O objetivo desse pré-teste era servir como linha de base para as relações que posteriormente seriam ensinadas.

\section{Procedimento Geral}

O procedimento para coleta de dados se dividiu em duas fases. A primeira fase do experimento se constituiu de um procedimento de escolha de acordo com o modelo (MTS) e a segunda fase de um procedimento de escolha sob esquemas concorrentes com encadeamento (Catania, 1999).

\section{Fase 1- Procedimento de Escolha de Acordo com o Modelo (MTS)}

Cada tarefa de MTS iniciou com a apresentação de um estímulo modelo, centralizado na metade superior do monitor do computador e da instrução "clique". Quando o participante clicava com um mouse no estímulo apresentado, o programa apresentava três estímulos de comparação na metade inferior da tela de forma equidistante (esquerda, centro, direita) e outra instrução "clique" era disponibilizada. A escolha do estímulo de comparação era realizada clicando-se sobre o estímulo. Após a resposta de escolha, a tentativa era finalizada com a apresentação de consequências programadas para respostas definidas, arbitrariamente, como corretas ou incorretas. Respostas definidas como corretas eram seguidas de uma animação apresentada no monitor do computador (moeda entrando no cofre) e respostas definidas como incorretas eram seguidas de uma tela escura por 2 segundos.

Todas as tentativas eram apresentadas de forma semi-randômica, sendo que não eram permitidas mais do que duas apresentações sucessivas da mesma relação (ex., A1B1) e também não eram permitidas apresentações sucessivas de duas comparações corretas na mesma posição na tela do computador (esquerda, centro, direita). O critério para estabilidade de resposta e término de cada fase de ensino foi definido como a emissão de $100 \%$ de respostas corretas em uma sessão. Caso o participante não atingisse o critério (quando era demonstrada perda de repertório, formação de classes não programadas ou respostas aleatórias), a instrução adicional "O porquinho indica que você está certo" era fornecida. Todas as sessões foram realizadas em um único dia. A Figura 1 ilustra a rede de relações ensinadas e testadas durante a Fase 1.

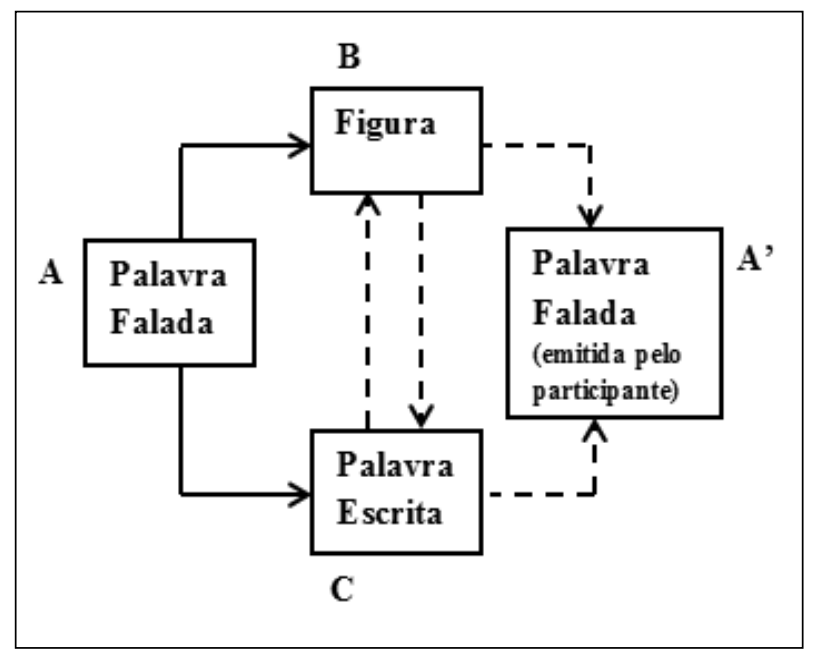

Figura 1. Rede de relações ensinadas e testadas no procedimento de escolha de acordo com o modelo. As setas contínuas representam as relações ensinadas e as pontilhadas representam as relações testadas.

Ensino da Relação $A B$. Inicialmente foram ensinadas as relações entre palavras faladas (A) e figuras correspondentes (B). A primeira tentativa foi iniciada com a apresentação do estímulo-modelo (A1), seguida da instrução "clique". Após a resposta do participante, três estímulos de comparação (B1, B2 e B3) foram apresentados na parte inferior da tela, seguidos da instrução: "clique". Após a escolha do estímulo comparação e apresentação das consequências, as demais tentativas foram apresentadas, seguindo-se o mesmo padrão. Uma sessão deste tipo constava de 18 tentativas, sendo três de cada uma das possíveis relações AB (A1-B1, A2-B2, A3-B3). Todas as tentativas $(100 \%)$ foram seguidas de consequência programadas e apresentação imediata de nova tentativa. $\mathrm{O}$ critério de ensino foi de $100 \%$ de acertos em uma sessão. 
Ensino da Relação $A C$. Em seguida, foram ensinadas as relações entre as palavras faladas (A) e as palavras impressas $(C)$, de maneira análoga ao ensino da relação $\mathrm{AB}$, em que os estímulos do conjunto $\mathrm{B}$ eram substituídos pelos estímulos do conjunto $\mathrm{C}$.

Ensino das Relações $A B$ e AC. Após alcance de critério, foi apresentada uma tarefa de ensino das relações $\mathrm{AB}$ e $\mathrm{AC}$ em conjunto, de maneira análoga ao ensino da relação $\mathrm{AB}$. Uma tarefa desse tipo constava de 18 tentativas, sendo nove da relação $\mathrm{AB}$ e nove da relação $\mathrm{AC}$. As tentativas foram seguidas de consequência programadas e apresentação imediata de nova tentativa. O critério permaneceu $100 \%$ de acerto para uma sessão.

Ensino das Relações AB e AC com $0 \%$ de Reforço. Essa fase era idêntica à fase anterior e nenhuma instrução nova foi fornecida, porém as tentativas não eram seguidas da animação ou da tela escura por 2 segundos, e sim pela apresentação imediata de uma nova tentativa. O critério para as tarefas de teste foi de $94 \%$ de acertos em uma sessão.

Testes das Relações $B C$ e CB. Após o ensino e teste das relações $\mathrm{AB}$ e $\mathrm{AC}$, foram testadas as relações emergentes entre os estímulos do conjunto B e do conjunto C. Uma sessão desse tipo constava de 18 tentativas, sendo três tentativas das relações $\mathrm{AB}$ previamente ensinadas (A1-B1; $\mathrm{A} 2-\mathrm{B} 2$, e A3-B3); outras três tentativas das relações $\mathrm{AC}$ também previamente ensinadas (A1-C1; A2-C2, e A3-C3); seis tentativas das relações $\mathrm{BC}(\mathrm{B} 1-\mathrm{C} 1$; $\mathrm{B} 2-\mathrm{C} 2$; $\mathrm{B} 3-\mathrm{C} 3)$; e mais seis tentativas das relações $\mathrm{CB}(\mathrm{C} 1-\mathrm{B} 1$; $\mathrm{C} 2-\mathrm{B} 2$; $\mathrm{C} 3-$ B3). Todas as escolhas foram seguidas pela apresentação imediata de uma nova tentativa.
Testes das Relações BA' e CA'. Em seguida, foi testada a resposta oral (A'), na presença das figuras (B) e na presença das palavras impressas (C). Nessas sessões, foi apresentada a instrução oral "Fale o nome" assim que a figura ou a palavra impressa aparecesse na tela do computador. Uma sessão desse tipo constava de 18 tentativas, sendo três tentativas das relações $\mathrm{AB}$; três tentativas das relações $\mathrm{AC}$; seis tentativas das relações $\mathrm{BA}^{\prime}$ (B1A'1, B2A'2, B3A'3); e mais seis tentativas das relações CA' (C1A'1, C2A'2, C3A'3). Todas as escolhas foram seguidas pela apresentação imediata de uma nova tentativa.

\section{Fase 2 - Avaliação de Preferência por Tarefas}

Esta fase tinha como objetivo identificar preferência por tarefas com figuras ou com palavras impressas. $\mathrm{O}$ comportamento de escolha foi observado sob esquemas de reforçamento concorrentes com encadeamento, como mostra a Figura 2. O participante tinha à sua frente dois botões na mesma cor vermelha, Botão 1 (lado esquerdo) e Botão 2 (lado direito). Ao início de cada tentativa, o participante recebia a instrução: "Aponte qual você quer". Nos elos iniciais, respostas ao Botão 1 em esquema de reforçamento de Razão fixa 1 (FR1), foram seguidas da apresentação de um dos estímulos do Conjunto B (Alternativa 1 - nomeação de figuras do Conjunto $\mathrm{B}$, apresentadas de forma randômica), no respectivo elo terminal. Respostas ao Botão 2 em esquemas de reforçamento de Razão fixa 1 (FR1), foram seguidas da apresentação de um dos estímulos do Conjunto C (Alternativa 2 - nomeação de palavras impressas do Conjunto $\mathrm{C}$, apresentadas de forma randômica), no respectivo elo terminal, conforme Figura 2.

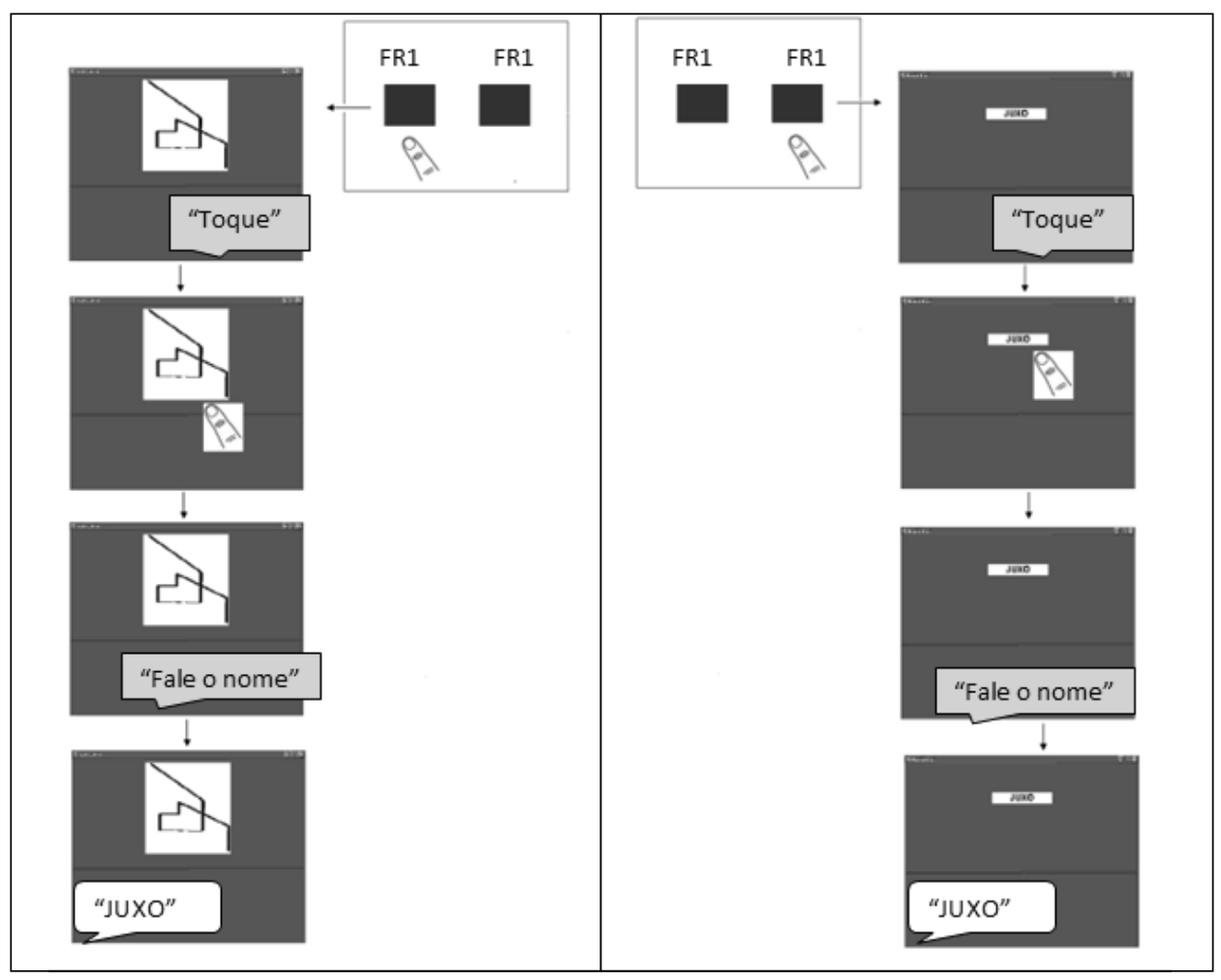

Figura 2. Esquemas da tarefa de escolha sob esquema de reforço concorrente com encadeamento. 
Guimarães, L. M., Escobal, G. \& Goyos, C. (2014). O Papel da Função Reforçadora das Relações Verbais de Tato e Textual, em Contexto de Equivalência de Estímulos, em Universitários.

A posição das alternativas nesse contexto era fixa. Após a apresentação do estímulo, o participante respondia oralmente aos estímulos: figura ou palavra impressa, e uma nova tentativa era apresentada. Uma sessão era composta por 20 tentativas. O critério para estabilidade de respostas de escolha e término dessa fase foi definido como a emissão de $75 \%$ ou mais respostas de escolha nos elos iniciais dos esquemas com preferência para uma das alternativas em duas sessões consecutivas. Quando o participante atingia o critério para desempenho nessa fase, a posição das alternativas: nomeação de figura e nomeação de palavra impressa era invertida.

\section{Procedimento para Registro e Análise de Dados e para Cálculo de Fidedignidade}

Os dados foram analisados de acordo com o delineamento experimental de sujeito único, tendo o participante como seu próprio controle (Tawney \& Gast, 1984). Os resultados de interesse na primeira fase do estudo foram: (a) identificar se os sujeitos aprenderam as relações $\mathrm{AB}$ e $A C$; (b) identificar se as relações $\mathrm{BC}$ e $\mathrm{CB}$ emergiram; (c) identificar se as relações BA' e CA' emergiram; (d) Comparar o desempenho em BA' e CA' nos testes de nomeação (tato e textual). $\mathrm{O}$ registro das respostas dos participantes foi realizado automaticamente pelo programa Mestre Libras, apenas as sessões de teste BA' e CA' foram gravadas em vídeo e submetidas a cálculo de fidedignidade.

Para a análise do comportamento de escolha, tanto do procedimento preliminar quanto da Fase 2, a variável dependente foi a taxa relativa de respostas de escolha: número de respostas no elo inicial correspondente aos Botões 1 e 2 (direita e esquerda, respectivamente) e as alternativas BA' e CA' (tato e textual, respectivamente), dividido pelo número de respostas no elo inicial correspondente a alternativas, representada pela fórmula: Taxa relativa de respostas $=\mathrm{R} 1 / \mathrm{R} 1+\mathrm{R} 2$ ou R2/R1+R2 (Fisher \& Mazur, 1997). Os dados das avaliações de preferência foram coletados a partir de um protocolo de registro observacional e registros em filme. Essas observações e registros foram submetidos ao cálculo de fidedignidade (Hall, 1974).

O cálculo de fidedignidade foi realizado pela experimentadora e por um observador independente treinado para esse fim e foi obtido a partir de $20 \%$ das tentativas, escolhidas ao acaso, considerando, para cada tentativa, o registro de uma concordância se o dado registrado pelo computador e o registrado pelos observadores independentes marcassem a mesma resposta como correta ou incorreta; quando os dados fossem diferentes, uma discordância era registrada. Uma concordância entre os observadores foi definida como a emissão correta do nome do estímulo durante as tarefas de nomeação e a escolha do Botão 1 ou do Botão 2 na tarefas de preferência por tarefas BA' $v s$. CA' (Fase 2). A fórmula utilizada para esse cálculo foi: número de concordância entre os dois observadores, dividido pelo número de concordância mais discordância, multiplicado por $100 \%$ (Hall, 1974). A média dos resultados do cálculo de fidedignidade foi de $98,5 \%$ de concordância.

\section{Resultados e Discussão}

Em seguida serão apresentados os resultados organizados pela ordem de exposição aos procedimentos da Fase 1 (MTS) e Fase 2 (Avaliação de Preferência por Tarefas), comparando-os com seus pré-testes correspondentes.

\section{Fase 1 - Escolha de Acordo com o Modelo (MTS)}

No pré-teste de respostas orais das figuras e palavras impressas, todos os participantes apresentaram repertório de leitura (textual; $\mathrm{CA}^{\prime}$ ) e nenhum demonstrou repertório de tato (BA'). Infere-se que isto possa ter acontecido em função dos participantes serem universitários e possuírem o repertório de leitura generalizada, o que pode ter gerado alguma fluência no desempenho sob a condição de leitura.

Todos os participantes alcançaram critério para a relação AC em apenas uma sessão, no entanto, os desempenhos no ensino da relação $A B$ variaram de um participante para o outro. Ricardo, por exemplo, alcançou critério para o ensino $\mathrm{AB}$ em apenas 36 tentativas, por outro lado, Lucas e Nara alcançaram critério em 126 tentativas cada um. No caso desses dois participantes, a imagem do porquinho apresentada após acerto para respostas consistentes pode não ter funcionado como reforçador para o ensino, sendo necessária instrução adicional para alcance de critério. Irene alcançou critério $\mathrm{AB}$ em 108 tentativas, Gabriel alcançou em 54 tentativas e Larissa em 72 tentativas.

Todos os participantes demonstraram a emergência das relações BC e CB. Depois de estabelecidas as relações de equivalência e demonstradas as propriedades de simetria e de transitividade, todos os participantes demonstraram desempenho de $100 \%$ nas tarefas de tato e textual. Ao contrário do relatado nos estudos de Elias et al. (2008), Sidman (1971), Sidman e Creason (1973) e Sidman et al. (1974), não se notou diferenças nos desempenhos dos participantes nas tarefas BA' e CA'. Esse resultado é consistente com o fato dos participantes universitários já possuírem repertório de leitura e generalizada e terem maior facilidade no ensino das relações de equivalência, considerando que os estudos citados anteriormente trabalharam com indivíduos com dificuldade intelectual sem repertório de leitura. Os resultados das tarefas de ensino, teste de tato e textual encontram-se na Figura 3.

\section{Fase 2 - Avaliação de Preferência por Tarefas}

Durante o pré-teste de preferência por figura ou palavra impressa, a maioria dos participantes não demonstrou preferência pelos estímulos. O participante Lucas demonstrou leve preferência por figura (65\% na primeira sessão e $75 \%$ na segunda sessão). Ao final do experimento, quatro dos participantes não demonstraram preferência por um tipo específico de tarefa, o que é consistente com o estabelecimento das relações de equivalência e com a indiferença no desempenho nos testes BA' e CA' que se seguiram ao estabelecimento das classes. A indiferença também poderia 
ser explicada de outra maneira em função da igualdade do valor reforçador entre as alternativas. Para avaliar os efeitos do tipo de reforçador e de sua magnitude nas condições estudadas seria necessário, por exemplo, acentuar essas características em uma das condições, o que poderia ser feito em novos estudos.
Não obstante, dois participantes demonstraram preferência por tarefas. Larissa demonstrou preferência por tarefas com palavras impressas ( $80 \%$ na primeira sessão e $80 \%$ na segunda sessão) e Lucas demonstrou preferência por tarefas com figuras ( $95 \%$ na primeira sessão e $90 \%$ na segunda sessão), conforme Figura 4.

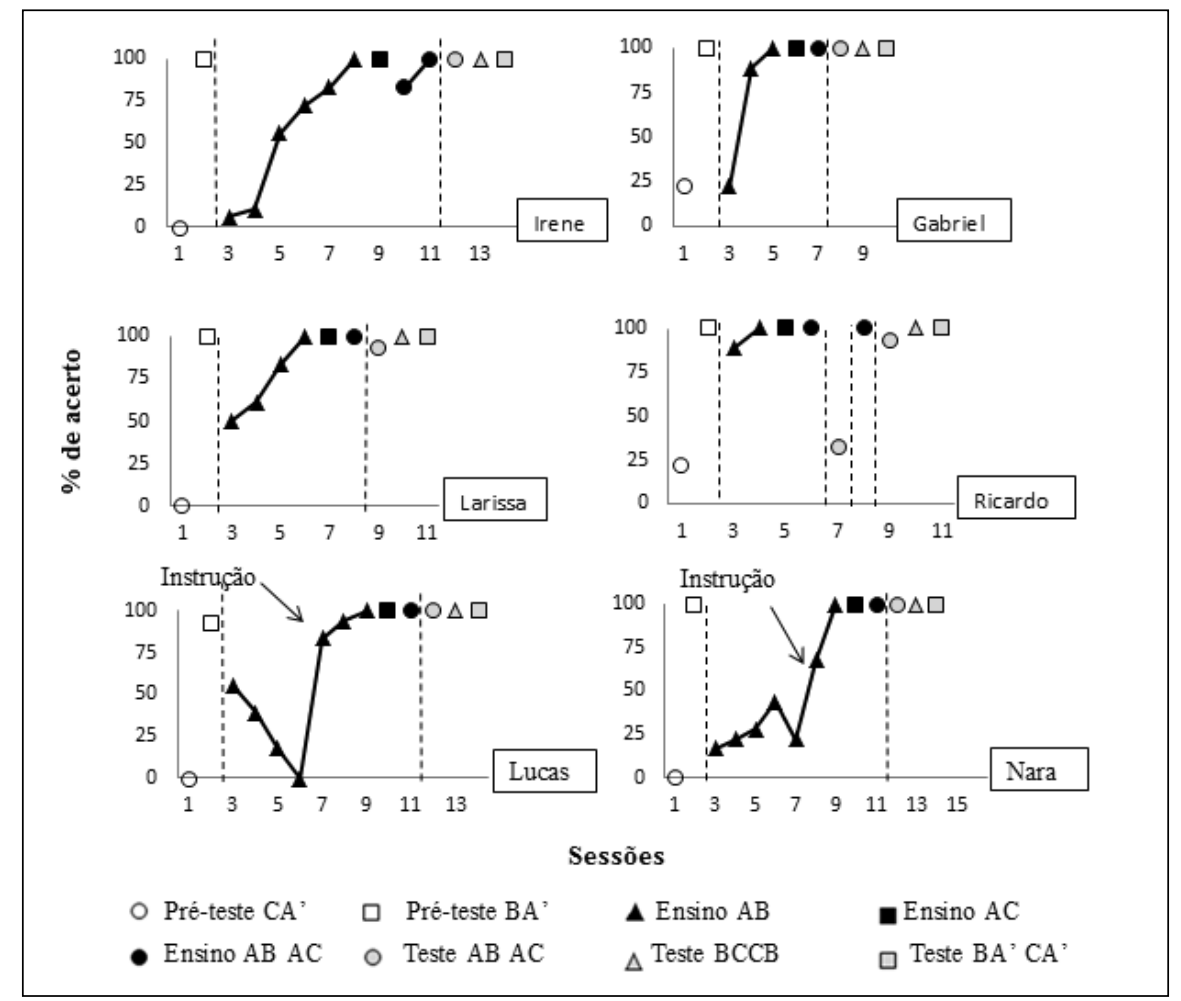

Figura 3. Desempenho dos participantes nos pré-testes das relações BA' CA', no ensino e teste das $\mathrm{ABAC}$, e teste das relações emergentes $\mathrm{BCCB}$ e BA' CA'.

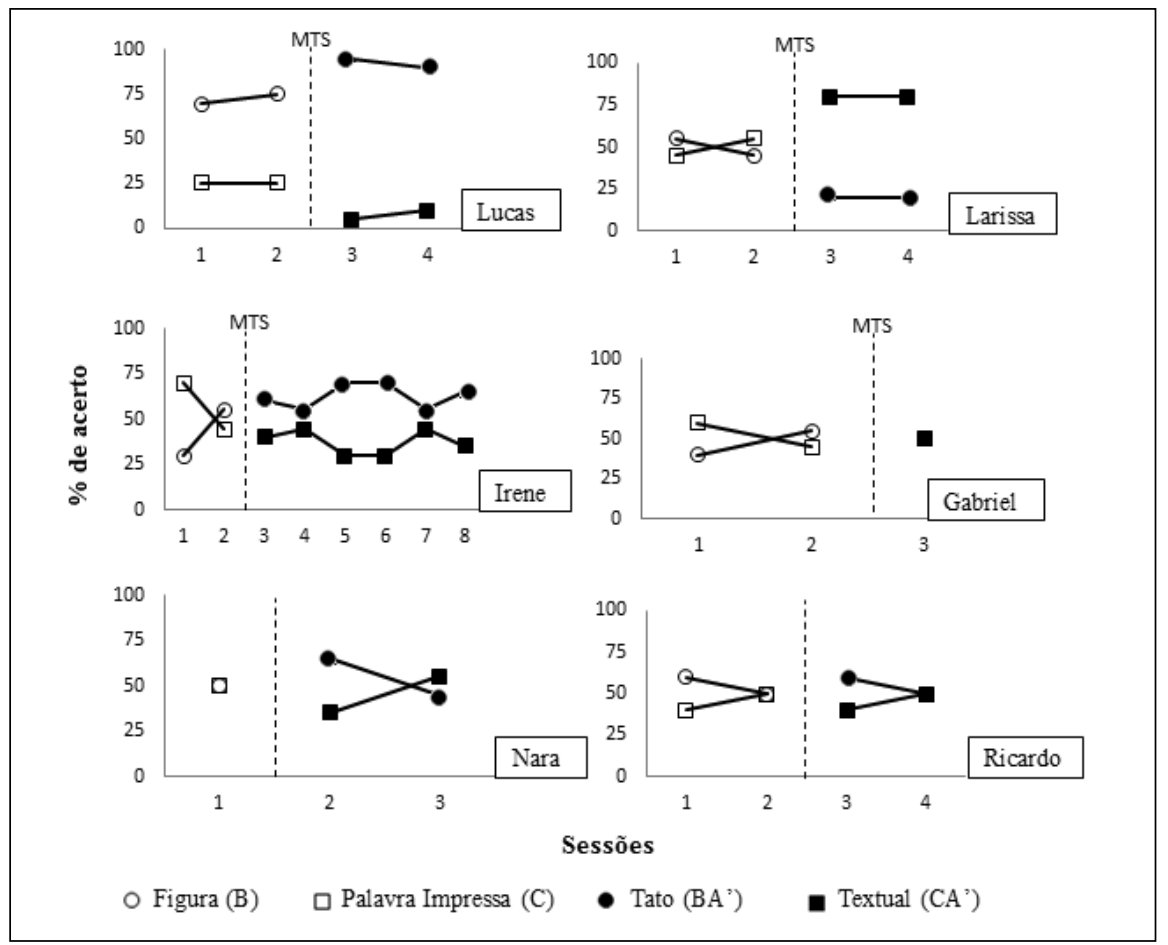

Figura 4. Desempenho dos universitários na avaliação de preferência B vs. C e por tarefas BA'vs. CA'. 
Apesar de Larissa não demonstrar preferência por figuras e palavras impressas durante o pré-teste, após o estabelecimento das relações de equivalência, ela passou a demonstrar preferência por tarefas com palavras impressas. Já no caso de Lucas, na avaliação de preferência por figuras e palavras impressas, o participante já demonstrava uma tendência a preferência por figuras, o que aumentou ainda mais na avaliação de preferência por tarefas com esses estímulos (BA' e CA'). A despeito de Lucas necessitar de instrução adicional, o que poderia fornecer um caráter aversivo à atividade, o participante ainda assim preferiu tarefas BA'. A preferência por figura pode ter influenciado a preferência final por tarefas BA' com figuras.

Mesmo não se notando diferenças importantes nas respostas orais frente a figuras e palavras impressas, dois participantes demonstraram preferência por tarefas: um por tarefas de tato e outro por tarefas textuais. Esse dado relativo à preferência desses participantes traz a tona ainda mais discussões acerca da preposição de Sidman (1994) de que a diferença entre membros de uma mesma classe é uma contradição. Esse estudo demonstra que os estímulos de uma mesma classe de equivalência podem possuir diferenças importantes relativas à preferência por tarefas que envolvem esses estímulos.

A preferência encontrada favorece a hipótese de que variáveis como características do próprio estímulo, como a familiaridade, por exemplo, podem estar influenciando a preferência. $\mathrm{O}$ fato de o estudo ter sido realizado com universitário, entretanto, é uma limitação importante já que a leitura generalizada apresentada por esses participantes pode ter influenciado o resultado. Novos estudos devem ser realizados com crianças com desenvolvimento típico e com atraso no desenvolvimento intelectual, sem repertório de leitura, no intuito de amenizar a variável história prévia com o estímulo.

Outra hipótese que pode ser levantada é que a dificuldade da tarefa pode ter funcionado como variável importante na preferência dos dois participantes. Tendo em vista que tarefas difíceis podem ser reforçadoras para determinados indivíduos e aversivas para outros. No entanto, novos estudos devem ser desenvolvidos para testar tais hipóteses.

$\mathrm{O}$ presente estudo quantificou os efeitos dos supostos estímulos reforçadores envolvidos na situação de escolha através do procedimento de esquemas concorrentes com encadeamento, o que reforça sua utilidade para estudos desta natureza (de Villiers, 1977).

\section{Considerações}

O presente estudo se propôs a avaliar o repertório de entrada e forneceu os subsídios para o planejamento de ensino direcionado às necessidades individuais. A análise das porcentagens de erros possibilitou a programação do ensino específico para as relações ausentes ou insuficientes. O uso do programa computacional Mestre Libras, da estrutura das tarefas de pré-teste, ensino e testes imediatos, para avaliar e ensinar mostrou-se eficiente, viável e permitiu que os participantes ficassem sob a influência quase estrita do conteúdo da tarefa, já que foi verificada a emergência de relações entre estímulos. Os resultados oferecem ainda evidências adicionais que confirmam a robustez do fenômeno de equivalência de estímulos com procedimento de ensino, que se reafirma como um método para a investigação sistemática para o ensino individual. Com base nessas considerações, as avaliações de preferência pelos estímulos experimentais e por tarefas de tato e textual propostos por esse estudo possibilitaram a quantificação de possíveis valores reforçadores envolvidos na situação de ensino, o que pode ser de extrema importância no desenvolvimento de procedimentos de ensino ainda mais eficazes.

\section{Referências}

Catania, A. C. (1999). Aprendizagem: Comportamento, linguagem e cognição (D. G. Souza, Trad.). Porto Alegre, RS: Artes Médicas.

Critchfield, T. S., \& Fienup, D. M. (2010). Using stimulus equivalence technology to teach about statistical inference in a group setting. Journal of Applied Behavior Analysis, 43, 437-462.

de Villiers, P. A. (1977). Choice in concurrent schedules and a quantitative formulation of the law of effect. In W. K. Honig \& J. E. R. Staddon (Eds.), Handbook of operant behavior (p. 233-287). Englewood Cliffs, NJ: Prentice Hall.

Elias, N. C., \& Goyos, C. (2010). Mestrelibras no ensino de sinais: Tarefas informatizadas de escolha de acordo com o modelo e equivalência de estímulos. In E. G. Mendes \& M. A. Almeida (Eds.), Das margens ao centro: Perspectivas para as politicas e práticas educacionais no contexto da educação especial inclusiva (Vol. 1, pp. 223-234). São Carlos, SP: Junqueira \& Marin.

Elias, N. C., Goyos, C., Saunders, M. D., \& Saunders, R. R. (2008). Teaching manual signs to adults with mental retardation using matching-to-sample procedures and stimulus equivalence. The Analysis of Verbal Behavior, 24, 1-13.

Escobal, G., \& Goyos, A. C. N. (2008). Análise das variáveis determinantes do comportamento de escolha entre alternativas de trabalho em adultos com deficiência mental. Revista Brasileira de Análise do Comportamento, 4, 71-87.

Escobal, G., Rossit, R. A. S., \& Goyos, A. C. N. (2010). Aquisição de conceito de número por pessoas com deficiência mental. Psicologia em Estudo (Maringá), 15, 467-475.

Fienup, D. M., Covey, D. P., \& Critchfield, T. S. (2010). Teaching brain-behavior relationships economically with stimulus equivalence technology. Journal of Applied Behavior Analysis, 43, 19-33.

Fisher, W. W., \& Mazur, J. E. (1997). Basic and applied research on choice responding. Journal of Applied Behavior Analysis, 30, 387-410.

Hall, R. V. (1974) Managing behavior - behavior modification: The measurement of behavior. Lawrence, KS: $\mathrm{H} \& \mathrm{H}$ Enterprises.

Hayes, S. C., Kohlenberg, B. S., \& Hayes, L. J. (1991). The transfer of specific and general consequential functions through simple and conditional equivalence relations. Journal of the Experimental Analysis of Behavior, 56, 119-137.

Horne, P. J., Hughes, J. C., \& Lowe, C. F. (2006). Naming and categorization in young children: IV. Listener behavior training and transfer of function. Journal of the Experimental Analysis of Behavior, 85, 247-273. 
Ribeiro, D. M., Elias, N. C., Goyos, C., \& Miguel, C. (2010). The effects of listener training on the emergence of tact and mand signs by individuals with intellectual disabilities. The Analysis of Verbal Behavior, 26, 65-72.

Rodríguez-Mori, M., \& Perez-Gonzáles, L. A. (2005). A simple procedure to teach condicional discriminations to children. Experimental Analysis of Human Behavior Bulletin, 23, 3-6.

Rossit, R. A. S., \& Goyos, C. (2009). Deficiência intelectual e aquisição matemática: Currículo como rede de relações condicionais. Revista Semestral da Associação Brasileira de Psicologia Escolar e Educacional, 13(2), 213-225.

Sidman, M. (1971). Reading and auditory visual equivalences. Journal of Speech and Hearing Research, 14, 5-13.

Sidman, M. (1994). Equivalence relations and behavior: A research story. Boston, MA: Authors Cooperative.

Sidman, M. (2000). Equivalence relations and the reinforcement contingency. Journal of the Experimental Analysis of Behavior, 74, 127-146.

Sidman, M., \& Cresson, O. (1973). Reading and crossmodal transfer of stimulus equivalences in severe retardation. American Journal of Mental Deficiency, 77, 515-523.

Sidman, M., Cresson, O., \& Willson-Morris, M. (1974). Acquisition of matching to sample via mediated transfer. Journal of the Experimental Analysis of Behavior, 22, 261-273.

Sidman, M., \& Tailby, W. (1982). Conditional discrimination vs. matching to sample: An expansion of the testing paradigm. Journal of the Experimental Analysis of Behavior, 37, 5-22.

Skinner, B. F. (1957) Verbal behavior. Englewood Cliffs, NJ: Prentice-Hall.

Stromer, R., Mackay, H. A., \& Stoddard, L. T. (1992). Classroom applications of stimulus equivalence technology. Journal of Behavioral Education, 2, 225-256.

Tawney, J. W., \& Gast, D. L. (1984). Single subject research in special education. Columbus, OH: Charles E. Merril. 